\title{
Comportement hydrodynamique de mélanges kaolinite-montmorillonite en conditions sodiques. Influence de la salinité
}

\author{
S Charpentier * \\ Laboratoire de sciences des sols et des substrats, ENSH-ENITHP, 2, rue Le Nôtre, F-49045 Angers cedex, France
}

(Reçu le 29 août 1996 ; accepté le 20 mars 1997)

\begin{abstract}
Summary - Hydrodynamic behaviour of sodic montmorillonite-kaolinite mixtures. Effects of salinity level. Mixtures of kaolinite and montmorillonite whose montmorillonite content ranged from 0 to $100 \%$ were obtained from suspensions of pure clays in 0.05 and $0.5 \mathrm{M} \mathrm{NaCl}$ solutions. Mixtures were then overconsolidated under saturated conditions up to $100 \mathrm{kPa}$. A mathematical model of internal stress relaxation in the overconsolidated domain allowed us to calculate the permeability for each sample. For media prepared at $0.05 \mathrm{M}$, results showed that at approximately $30 \%$ of montmorillonite, there was a threshold where the permeability of the medium was the permeability of the pure montmorillonite. Relations between void ratio and swelling indexes versus montmorillonite content presented the same singularity for this value. Conversely, variations of these properties are proportional to the montmorillonite content for media obtained in $0.5 \mathrm{M}$ solution. A rapid observation and treatment of images obtained by scanning electron microscopy led us to propose the hypothesis that when the content of montmorillonite reaches the value of $30 \%$, tactoïds obtained in diluted and sodic conditions realise a continous lattice that can impose the permeability of the montmorillonite to the whole medium.
\end{abstract}

montmorillonite / kaolinite / permeability / salinity

Résumé - Des mélanges de kaolinite et de montmorillonite dont les teneurs variaient entre 0 et $100 \%$ de montmorillonite ont été obtenus par mise en suspension des minéraux dans des solutions 0,5 ou $0,05 \mathrm{M}$ de $\mathrm{NaCl}$. Les mélanges ont ensuite été consolidés en conditions saturées jusqu'à une sollicitation de $100 \mathrm{kPa}$. Un modèle simple de relaxation de la contrainte dans le domaine consolidé en fonction du temps a permis de calculer les valeurs de la perméabilité intrinsèque pour chacun des échantillons. Pour les milieux préparés à $0,05 \mathrm{M}$, les résultats obtenus ont mis en évidence aux environs de $30 \%$ de montmorillonite un seuil à partir duquel la perméabilité du milieu est entièrement définie par la perméabilité de la montmorillonite pure. Les relations entre indice de vide et indice de gonflement d'une part et teneur en montmorillonite d'autre part présentent aussi un point singulier pour la même teneur. À l'opposé, la variation de ces propriétés est continue avec la teneur des mélanges obtenus à $0,5 \mathrm{M}$. Une étude rapide d'images obtenues au microscope à balayage a permis d'émettre l'hypothèse que, dès $30 \%$ de montmorillonite, les tactoödes obtenus en milieu dilué et sodique peuvent déjà former un réseau continu imposant la perméabilité de la montmorillonite à l'ensemble du milieu.

montmorillonite / kaolinite / perméabilité / salinité

\footnotetext{
* Correspondance et tirés à part

Tél : (33) 0241225420 ; fax : (33) 0241731557 ; courriel : sylvain.charpentier@angers.inra.fr
} 


\section{INTRODUCTION}

L'étude de l'écoulement de l'eau à travers les milieux argileux a suivi essentiellement deux directions complémentaires. La première est liée à la géométrie porale et à l'organisation. Ses résultats concernent les fortes teneurs en eau et sont avant tout dus à l'exploitation de la loi de Darcy. Sur des sols en place, des travaux récents (Cabidoche et Voltz, 1995) ont mis en évidence que la lenteur des transferts d'eau au niveau de l'agrégat ou du massif dans les vertisols à forte teneur en argiles gonflantes était en grande partie à l'origine des déformations non uniformes de ces sols à l'échelle du profil. Au-delà de ces situations particulières où la teneur en smectites peut dépasser $50 \%$ la présence de phases argileuses gonflantes peut jouer un rôle important sur le fonctionnement hydrique du milieu avec des proportions beaucoup plus faibles. Depuis Norrish et Quirk (1954) et Quirk et Schofield (1955), de nombreux travaux ont confirmé l'importance de la nature du cation compensateur et de la salinité du milieu sur le gonflement et la perméabilité des matériaux smectitiques.

La seconde réunit tous les travaux qui tentent d'appréhender les propriétés chimiques de l'eau au voisinage des feuillets argileux et qui s'appliquent aux faibles teneurs en eau. Les travaux de Mamy (1968) sur les incidences de la structure ou des propriétés diélectriques du film d'eau sur l'entrée d'eau entre les feuillets ou ceux de Low (1976) sur les estimations de la viscosité au voisinage du solide en sont des exemples. Cases et François (1982) limitent l'effet du champ de surface du solide dans la solution au maximum à quatre couches de molécules d'eau avec un fort pourcentage de la surface occupé par une couche bimoléculaire (Cases et al, 1992) lorsque l'humidité relative est de 0,9 soit un potentiel de l'eau d'environ -150 MPa. Il est aussi acquis que, dans certains milieux argileux à teneur en eau moyenne ou élevée, toute l'eau n'est pas concernée par les phénomènes d'écoulement et ceci pas uniquement pour des raisons de géométrie porale, mais aussi pour des raisons physicochimiques. Des approches électrocinétiques (Cuisset, 1979) ou rhéologiques (Keren et Singer, 1988 ; Keren, 1989), effectuées sur des suspensions, constituent une première approche des propriétés physicochimiques de l'eau dans les milieux argileux à forte teneur en eau. Manifestement, les conditions physicochimiques de l'ensemble solution-soluté -solide ont un effet prépondérant sur l'extension de la zone d'influence du solide et les propriétés d'écoulement de l'eau. Pour certains milieux argileux, plus la solution sera sodique et/ou diluée, plus l'influence de la surface argileuse chargée sera importante dans le liquide.

En ce qui concerne l'organisation des particules et les relations vis-à-vis de l'eau et de ses mouvements, kaolinite et montmorillonite ont des comportements opposés, particulièrement aux fortes teneurs en eau, lorsque les conditions sont sodiques et peu concentrées. De part et d'autre d'une limite de potentiel osmotique située aux alentours de $-2000 \mathrm{~J} \cdot \mathrm{kg}^{-1}$ (pression osmotique de $2 \mathrm{MPa}$; Charpentier, 1991), il y a réorganisation réversible des particules primaires de montmorillonite en quasicristaux ou en tactoïdes. Le milieu passe alors d'une organisation intergranulaire «type château de carte " à une organisation de «type alvéolaire » (Tessier, 1984).

Parallèlement à ces études sur des minéraux relativement purs et bien caractérisés, seuls quelques travaux ont tenté d'approcher le comportement de milieux polycristallins. Nous citerons ceux de Kenney en 1967 (in MüllerVonMoos et Loken, 1989) qui, en recherchant une relation entre la distribution granulométrique et les propriétés plastiques de mélanges argileux, avait étudié le comportement en cisaillement de mélanges sable - kaolinite d'une part et sablemontmorillonite d'autre part. Si la variation des propriétés apparaissait continue pour les mélanges à base de kaolinite, la chute était brutale pour les mélanges à base de montmorillonite et ne variait plus dès que le taux d'argile avait atteint $30 \%$.

L'étude des transferts d'eau à l'échelle de l'agrégat fonctionnel dans les milieux à forte teneur en argile représente une des approches dans la continuité à trouver entre d'une part l'étude du comportement mécanique ou physicochimique de matériaux simples dans des conditions parfaitement contrôlées et d'autre part l'étude du fonctionnement des sols riches en matériaux gonflants. En conditions naturelles, les agrégats de ces sols fonctionnent dans le domaine consolidé : ils sont en effet soumis à des cycles annuels de sollicitations dont les valeurs se répètent d'une année à l'autre en n'imposant que rarement de nouvelles conditions susceptibles de provoquer une évolution de la consolidation.

En mécanique des sols, l'étude du comportement d'un sol sous sollicitation externe est essentiellement représentée par l'étude de la consolidation. Que l'application de la sollicitation soit uniaxiale (œdomètre) ou triaxiale (essai triaxial), l'interprétation des courbes de consolidation est 
l'outil analytique privilégié de cette application de la mécanique des milieux continus. En revanche, l'étude des déformations en gonflement-retrait dans le domaine surconsolidé est moins courante.

D'un point de vue général, les milieux argileux aux fortes teneurs en eau peuvent être considérés comme des matériaux élastoplastiques à écrouissage non linéaire. Le comportement de ces milieux est illustré par la figure 1 (Mieussens et al, 1985 ; Bradford et Gupta, 1986) qui représente la courbe typique d'un essai de compressibilité œdométrique. Dans ces essais, sollicitation et déformation de l'échantillon sont uniaxiales et les relations contrainte-déformation peuvent être représentées par des diagrammes d'indice de vide $e$ en fonction de la pression $p$ appliquée sur l'échantillon et considérée comme transmise intégralement dans le milieu lorsque le système est à l'équilibre. Schématiquement, ils présentent des branches orientées selon deux directions parallèles aux droites $(\mathrm{c})$ ou $(\mathrm{g})$ qui se coupent au point $\mathrm{M}$ correspondant à une charge $p_{\mathrm{p}}$. Cette valeur représente assez bien la contrainte maximale que l'échantillon a subi et pourrait matérialiser une des composantes de ce que l'on appelle « l'histoire du matériau ». La direction (c) représente une consolidation du milieu et sa pente est appelée
Cc, « indice de compression »; elle correspond à l'application de contraintes que le matériau n'a encore jamais subies. La direction (g) correspond au domaine surconsolidé où les valeurs de contrainte appliquée sont inférieures à $p_{\mathrm{p}}$. Les cycles de chargement-déchargement y sont à peu près réversibles et, par comparaison aux matériaux métalliques, on peut parler d'un comportement élastique non linéaire. On désigne par $\mathrm{Cs}$, « indice de gonflement » la pente de la droite $(\mathrm{g})$. On a :

$$
\mathrm{C}_{\mathrm{cous}}=-\frac{\mathrm{d} e}{\mathrm{~d} \log p}
$$

D'autres coefficients expriment la même notion, en particulier le coefficient de compressibilité volumique $\mathrm{a}_{v}$ avec

$$
\mathrm{a}_{\mathrm{v}}=-\frac{\mathrm{d} e}{\mathrm{~d} p}=\frac{\mathrm{C}_{\mathrm{c}}}{2,3 p}
$$

Lorsqu'un volume argileux, saturé et déformable, est soumis à une sollicitation mécanique externe constante $p$, cette sollicitation va s'exercer sur les phases solide et liquide. La contrainte ainsi obtenue sur la phase liquide est à l'origine d'un échange d'eau avec l'extérieur et si la perméabilité du solide est limitée, cet échange est plus ou moins long dans le temps. Il existe donc

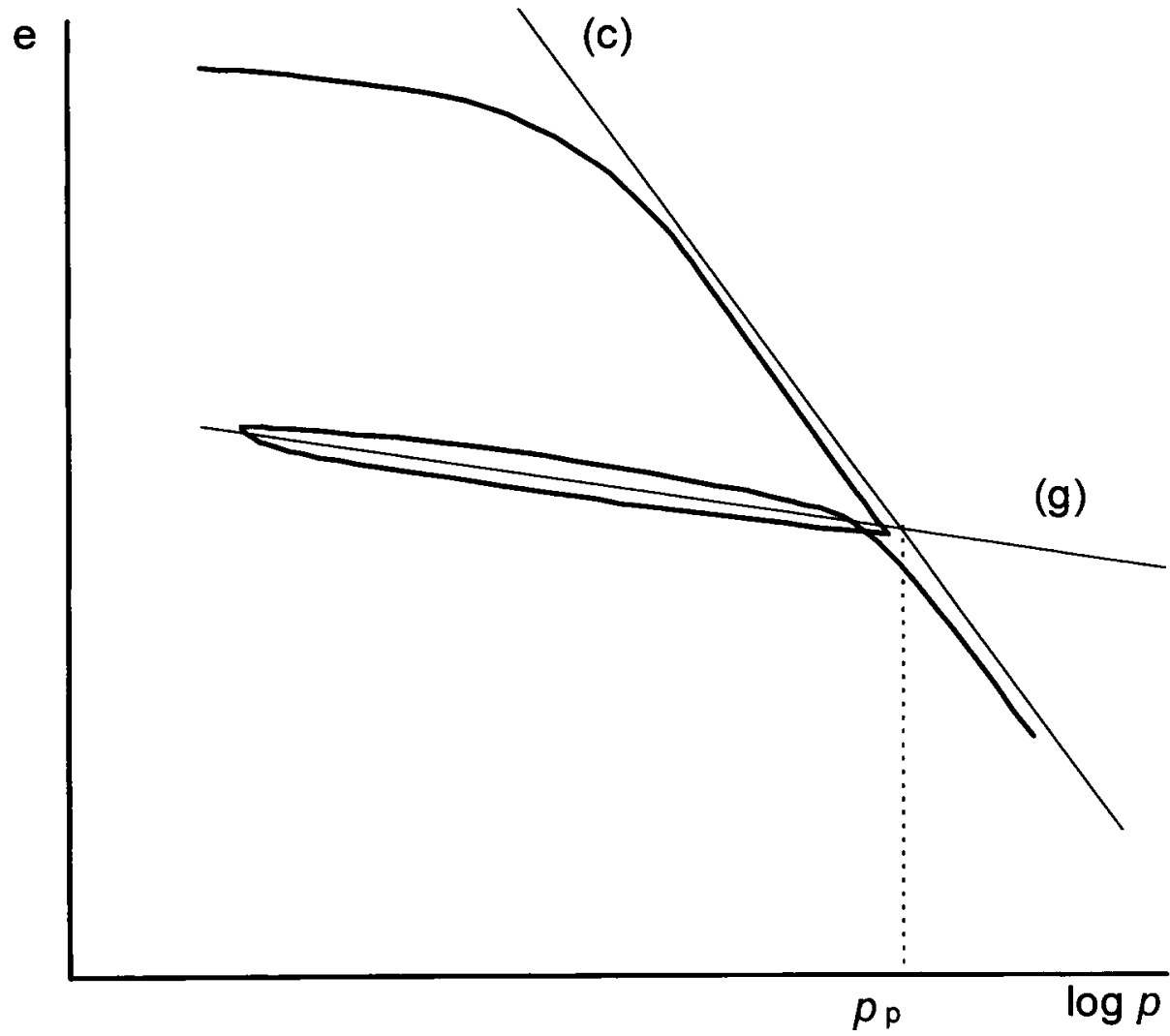

Fig 1. Essai de compressibilité cedométrique. 
deux contraintes, l'une que l'on appellera $p$ ' sur le solide et l'autre $p_{\mathrm{w}}$ sur la phase liquide qui sont des variables du temps entre l'équilibre qui existait avant l'application de la sollicitation et l'évolution vers un nouvel état d'équilibre.

Les travaux présentés dans cet article ont eu comme objectif l'observation du comportement sous sollicitation dans le domaine consolidé de mélanges argileux (kaolinite + montmorillonite) en milieu sodique. Deux niveaux de concentration de la phase liquide ont permis de distinguer les milieux où les montmorillonites sont organisées en tactoïdes des milieux où elles sont sous forme de quasicristaux. Les paramètres étudiés pour les différents milieux ont été :

- les indices de vide à l'équilibre ;

- le retrait-gonflement en relaxation de contrainte et l'indice de gonflement ;

- la perméabilité intrinsèque évaluée par traitement des courbes de retrait-gonflement en fonction du temps.

Une confirmation de certains résultats a été tentée avec l'analyse d'images obtenues au microscope électronique à balayage sur quelques échantillons.

\section{MATÉRIELS ET MÉTHODES}

\section{Mélanges et appareillage}

Pour cette approche, nous avons retenu deux argiles " modèles » de gisement pour lesquelles de nombreuses références existent déjà : la kaolinite de Saint Austell et la montmorillonite du Wyoming (Tessier, 1984). Avec ces deux argiles, une douzaine de mélanges ont été fabriqués avec des rapports massiques montmorillonite/kaolinite variant de 0 à $100 \%$. Ces mélanges ont été mis en suspension dans des solutions de $\mathrm{NaCl}$ de concentration 0,05 et 0,5 molaire. Les milieux ainsi obtenus contiennent trois types de particules :

- des cristallites et des quasicristaux pour les mélanges à $0,5 \mathrm{M}$;

- des cristallites et des tactoïdes pour les mélanges à $0,05 \mathrm{M}$.

Les mélanges récupérés après décantation ont été dans un premier temps consolidés sous une sollicitation de $100 \mathrm{kPa}$. Les principales déterminations, indice de vide, indice de gonflement-retrait et mesures en relaxation ont été effectuées en retrait entre 3,16 et $10 \mathrm{kPa}$ dans le domaine consolidé à $100 \mathrm{kPa}$. Le montage utilisé pour la consolidation et les mesures de retrait-gonflement est composé d'un dispositif de filtration de marque Sartorius (type SM 16510/11) auquel on adjoint une membrane cylindrique en latex de $0,1 \mathrm{~mm}$ d'épaisseur, de $30 \mathrm{~mm}$ de diamètre de 100 $\mathrm{mm}$ de longueur et fermée à l'une de ses extrémités (fig 2). Le montage est installé en évitant tout emprisonnement d'air dans le système (Charpentier, 1991). Les filtres utilisés ont une porosité de $0,45 \mu \mathrm{m}$ et sont toujours restés imperméables aux particules de solide. Le volume inférieur est en communication avec un réservoir externe maintenu à la pression atmosphérique et dont les pesées permettront de mesurer les entrées et les sorties de solution.

Une fois le système mis en place, les mélanges sont soumis à des sollicitations externes par application d'une pression pneumatique sur l'extérieur de la membrane. Certaines opérations de consolidation se sont avérées très longues particulièrement lorsque le taux

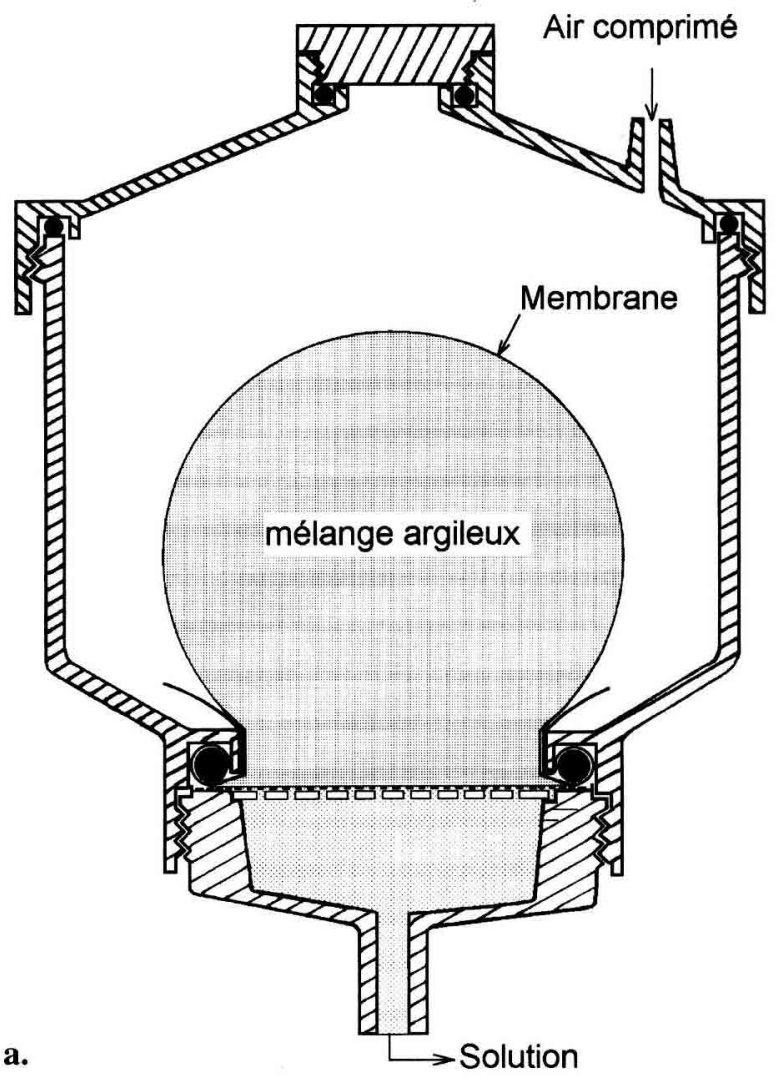

b.

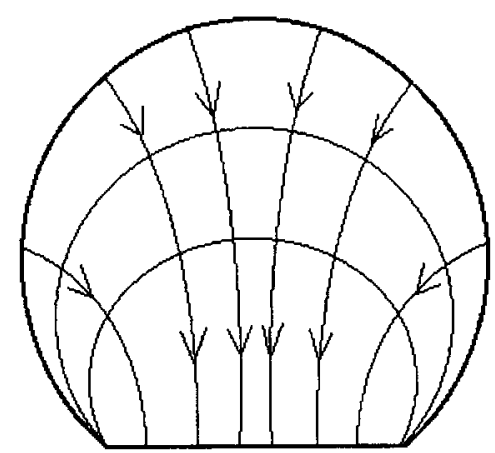

Fig 2.a. Montage expérimental et b. figuration des lignes de flux et des lignes équipotentielles. 
de montmorillonite est élevé. Le temps mis pour obtenir des variations relatives négligeables et une valeur supposée très proche de l'équilibre sous sollicitation maintenue constante a quelquefois atteint plusieurs mois. La souplesse et le coût réduit du système ont permis une installation en batterie et le traitement simultané de plusieurs suspensions. Comparativement à un œdomètre classique, ce montage supprime une grande partie des frottements de l'appareillage (pistoncylindre ou échantillon-cylindre) et permet de travailler à des niveaux de sollicitation mécanique peu élevés.

Certains mélanges ont été observés au microscope. Les prélèvements ont été faits au centre des massifs obtenus à la fin des opérations de consolidation. L'observation a été faite sur une surface obtenue après fracture de l'échantillon congelé à partir de la méthode décrite par Chenu et Tessier (1995). Les prises de vues ont été directement numérisées sur microscope électronique à balayage Philips. Les traitements numériques ont été réalisés à l'aide du logiciel de traitement d'image Visolog (version 4.1.3.) pour Microsoft Windows.

\section{Théorie et solution analytique simple de l'équation de consolidation}

La formulation mathématique générale des phénomènes transitoires de mouvement d'eau dans un milieu déformable dérive de la combinaison de plusieurs équations :

- l'équation de bilan appliquée à la conservation de la phase liquide,

$$
\frac{\mathrm{d} \theta_{w}}{\mathrm{~d} t}=-\operatorname{div}\left(\theta_{w} \overrightarrow{v_{w}}\right)
$$

où $\theta_{w}$ est la teneur volumique en eau et $\vec{v}_{w}$ la vitesse de la phase liquide dans un repère absolu ;

- la vitesse de Darcy sous sa forme généralisée,

$$
\vec{v}_{D}=\frac{\mathrm{k}}{\theta_{\mathrm{w}} \cdot \eta} \operatorname{grad}\left(p_{w}\right)
$$

qui représente la vitesse du liquide par rapport au solide. $\eta$ est le coefficient de viscosité dynamique. $k$, généralement appelé perméabilité intrinsèque, est un coefficient qui ne dépend que du solide et de l'indice des vides; ses dimensions sont celles d'une surface (Costet et Sanglerat, 1981). L'hypothèse de la proportionnalité entre la vitesse de Darcy et le gradient de potentiel permet d'obtenir une équation aux dérivées partielles à coefficient constant. Elle doit cependant être considérée comme une première approximation dés que le milieu devient riche en smectites où les phénomènes électocinétiques induits par l'écoulement peuvent devenir importants (Gras, 1988).

L'étude des mouvements d'eau dans les milieux déformables pose le problème du référentiel. L'équation aux dérivées partielles obtenue dans un repère unique à partir de ces relations est compliquée dans le cas général et seuls les cas particuliers de déformations monodirectionnelles verticales ont été abondamment traités. L'approche de Terzaghi dans les sols saturés en eau en est un exemple. Le postulat de Terzaghi considère qu'en conditions saturées le chargement total $p$ est additivement partagé entre la contrainte sur le solide $p$ ' appelée contrainte effective et la contrainte $p_{\mathrm{w}}$ sur le liquide qui représente la pression hydrostatique de cette phase :

$$
p=p^{\prime}+p_{\mathrm{w}}
$$

Si $p$ est maintenu constante, en tout endroit du système, on peut écrire :

$$
\mathrm{d} p^{\prime}=-\mathrm{d} p_{\mathrm{w}}
$$

En introduisant le coefficient de compressibilité $a_{v}$ on obtient la relation :

$$
\mathrm{d} e=-\mathrm{a}_{v} \cdot \mathrm{d} p^{\prime}=\mathrm{a}_{v} \cdot \mathrm{d} p_{\mathrm{w}}
$$

Si on admet que les déformations sont faibles, ce qui est particulièrement vrai pour les phénomènes de gonflement-retrait dans le domaine surconsolidé, et que les variations de la perméabilité intrinsèque sont aussi très limitées pendant la relaxation ( $\mathrm{k}$ constant), la combinaison de ces différentes relations (Vauclin, 1987), aboutit à l'équation classique de consolidation de Terzaghi (Gras, 1988) :

$$
\frac{\delta\left(p_{w}\right)}{\delta t}=\operatorname{div}\left(\frac{\mathrm{k} \cdot(1+\mathrm{e})}{\mathrm{a}_{\mathrm{v}} \eta} \overline{\operatorname{grad}}\left(p_{\mathrm{w}}\right)\right)
$$

où $C v=\frac{k \cdot(1+e)}{a_{v} \eta}$ est appelé coefficient de consolidation.

Le suivi dans le temps de la relaxation d'un massif argileux soumis à une sollicitation externe permet donc d'approcher la valeur de $\mathrm{k}$.

Dans le montage expérimental utilisé, l'échantillon est de géométrie sphérique (fig 2) et, lorsque le chargement externe augmente, le volume du système se réduit avec une forme générale qui reste sphérique. Les surfaces équipotentielles sont des sphères dont la trace sur le plan médian est représentée par un faisceau de cercles ayant pour points de base les extrémités du filtre. Ces points sont singuliers puisque les courbes équipotentielles s'y rejoignent. Sur le même plan, les lignes de courant qui matérialisent les mouvements de liquide et de solide sont représentées par une famille de cercles perpendiculaires au faisceau d'équipotentielles et dont les centres sont situés sur l'axe radical de ce faisceau. Sur ces lignes de courant, les vecteurs flux de liquide et flux de solide sont de même sens. L'échange de liquide se fait au niveau du filtre, surface circulaire plane. Contrairement à un système de symétrie sphérique où l'échange se ferait au niveau de la surface sphérique extérieure de l'échantillon, cette dernière est, dans le montage que nous avons utilisé, une surface de flux nul de liquide. Avec toutes ces caractéristiques, le système décrit ci-dessus est assez proche d'une déformation uniaxiale de type œdométrique. Nous avons donc retenu cette approche qui est simple 
et offre de nombreuses solutions analytiques. En déformation uniaxiale, l'équation [8] devient alors :

$$
\frac{\delta\left(p_{\mathrm{w}}\right)}{\delta t}=\mathrm{C}_{\mathrm{v}} \frac{\delta\left(p_{n}\right)}{\delta x^{2}}
$$

Des solutions analytiques à ces équations ont été développées dans de nombreux cas de figure (Houpeurt, 1975) : elles utilisent des variables réduites. Pour pouvoir interpréter rapidement les résultats du montage expérimental utilisé, nous avons donc choisi d'assimiler son principe au tassement uniaxial d'un massif de longueur 1 hermétiquement fermé à l'une de ses extrémités et en communication avec un réservoir de liquide à l'autre. Dans l'état initial, l'eau est à la pression $p_{w 1}$ dans le massif et dans le réservoir. Au temps $\mathbf{t}=0$, le potentiel de l'eau du massif est porté au potentiel $p_{\mathrm{w} 2}$ par application d'une sollicitation mécanique à l'échantillon. Le liquide va s'écouler par la face en communication avec le réservoir et la différence de potentiel de l'eau entre le massif et le réservoir va progressivement décroître pour atteindre un nouvel équilibre à la pression $p_{w 1}$. Ce cas de figure est un problème de flux nul à distance finie (Houpeurt, 1975).

Les variables réduites utilisées sont :

$$
x_{\mathrm{R}}=\frac{x}{1} \quad t_{\mathrm{R}}=\begin{aligned}
& \mathrm{C}_{\mathrm{v}} t \\
& \mathrm{I}^{2}
\end{aligned} \quad P_{\mathrm{R}}=\frac{p_{\mathrm{w}}-p_{\mathrm{w} 1}}{p_{\mathrm{w} 2}-p_{\mathrm{w} 1}}
$$

Et l'équation 10 devient :

$$
\frac{\delta P_{\mathrm{R}}}{\delta t_{\mathrm{R}}}=\frac{\delta^{-} P_{\mathrm{R}}}{\delta x_{\mathrm{R}}^{2}}
$$

Les conditions aux limites sont :

$$
\begin{array}{lll}
x_{\mathrm{R}}=0 & P_{\mathrm{R}}=0 & \text { quel que soit } t_{\mathrm{R}} \\
x_{\mathrm{R}}=1 & \mathrm{~d} P_{\mathrm{R}} / \mathrm{d} x_{\mathrm{R}}=0 & \text { quel que soit } t_{\mathrm{R}} \\
t_{\mathrm{R}}=0 & P_{\mathrm{R}}=1 & \text { quel que soit } x_{\mathrm{R}} \# 0
\end{array}
$$

L'intégration de l'équation [11] s'effectue en admettant que la solution est une combinaison linéaire des solutions particulières, et en vérifiant les conditions aux limites. Le problème aboutit alors à celui d'un développement en série de Fourier. On en trouvera une solution détaillée dans Houpeurt (1975). Le calcul de la production cumulée réduite, rapport entre la quantité de liquide sortie (ou entrée) au temps $t$ et la quantité totale sortie (ou entrée) en fin de relaxation est un résultat intéressant. En effet, le montage expérimental que nous avons utilisé permet facilement de mesurer à tout instant ce volume de liquide. Le volume de solution échangée correspond au tassement ou retrait puisque la compression s'effectue en saturé. Nous trouvons plus judicieux d'utiliser le terme retrait volumique réduit symbolisé par $R$ et qui sera égal au rapport entre le retrait au temps $t$ et le retrait en fin de relaxation.

L'expression de la fonction $R=\mathrm{f}(t)$ prend la forme :

$$
R=1-\frac{8}{\pi^{2}} \sum_{0}^{\infty} \frac{1}{(2 \mathrm{~m}+1)^{2}} \cdot \mathrm{e}^{-(2 \mathrm{~m}+1)^{2} \cdot \pi^{2} \cdot t_{\mathrm{R}} / 4}
$$

et le retrait maximal n'est théoriquement obtenu que pour un temps infini. Numériquement, on démontre (Houpeurt, 1975) que pour $t_{\mathrm{R}}>0,2, R$ est très peu différent de :

$$
R=1-\frac{8}{\pi^{2}} \cdot \mathrm{e}^{-\pi^{2} t_{\mathrm{R}} / 4}=1-\frac{8}{\pi^{2}} \cdot \mathrm{e}^{-\pi^{2} \mathrm{c}_{\mathrm{v}} t / 41^{2}}
$$

Les courbes de consolidation $R=\mathrm{f}(t)$ permettent d'approcher la valeur de $\mathrm{Cv}$ qui est lié aux trois paramètres suivants : le coefficient de compressibilité $\mathrm{a}_{v} \mathrm{du}$ solide, la viscosité $\eta$ de la phase liquide et la perméabilité intrinsèque $\mathrm{k}$ du solide

\section{RÉSULTATS}

\section{Variations de l'indice de vide et de l'indice de gonflement}

Les valeurs d'indice de vide reportées sur la figure 3 sont les valeurs moyennes de l'indice de vide observé lors de la sollicitation en retrait de 3,16 à $10 \mathrm{kPa}$ dans le domaine consolidé à 100 $\mathrm{kPa}$. Pour les mélanges à $0,5 \mathrm{M}$, les indices de vide varient entre les valeurs extrêmes de 1,41 pour la kaolinite pure et 2,59 pour la montmorillonite pure et les mélanges intermédiaires sont bien alignés entre ces deux pôles. Avec les mélanges à $0,05 \mathrm{M}$, les indices de vide varient de 1,47 à 7,39 et sont organisés autour de deux directions principales. Les droites de régression obtenues à partir de ces deux familles de points se coupent à une teneur massique de montmorillonite d'environ $30 \%$.

Toutes proportions gardées, les allures des relations indice de gonflement-teneur en montmorillonite (fig 4) sont comparables à celles représentant l'indice de vide. Le même effet de mélange peut être observé aux environs de $30 \%$ de montmorillonite pour les milieux à $0,05 \mathrm{M}$.

\section{Variation de la perméabilité}

La relation [14] a été ajustée aux résultats expérimentaux en recherchant pour chaque opération une régression linéaire pour les couples de valeurs $\left(t, \operatorname{Ln}\left(\pi^{2}(1-R) / 8\right)\right)$. Quelques résultats sont reportés sur la figure 5 pour les milieux à $0,5 \mathrm{M}$ et la figure 6 pour les milieux à $0,05 \mathrm{M}$. Les courbes ajustées du modèle sont en trait plein pour les valeurs correspondant à $t_{\mathrm{R}}>0,2$ et en tiretés pour les valeurs de $t_{\mathrm{R}}<0,2$. Une lecture qualitative rapide des courbes des figures 5 et 6 permet de voir que le temps de mise en équilibre a pour ordre de grandeur: 
Fig 3. Relations indice de videteneur massique en montmorillonite pour les mélanges argileux

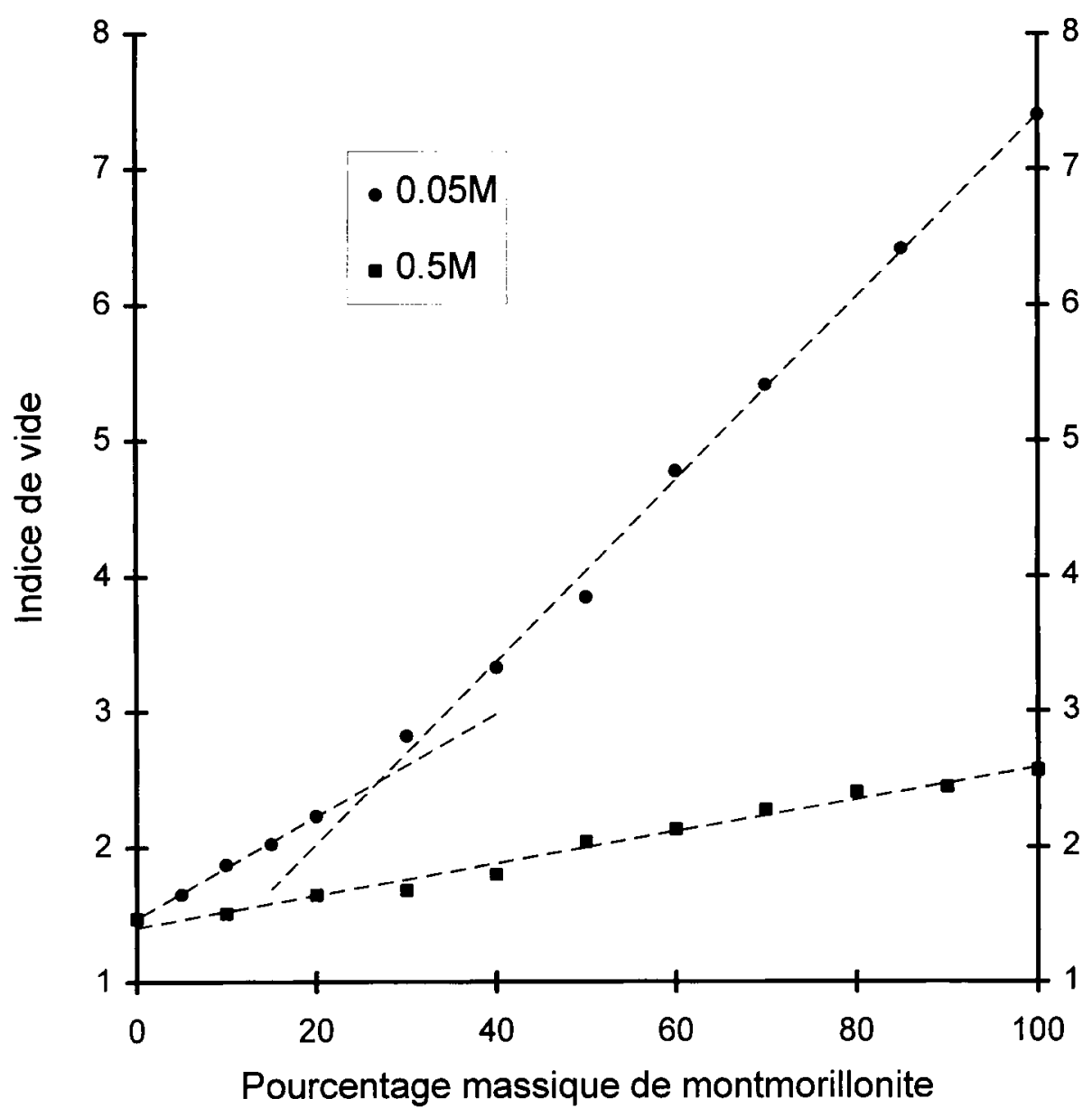

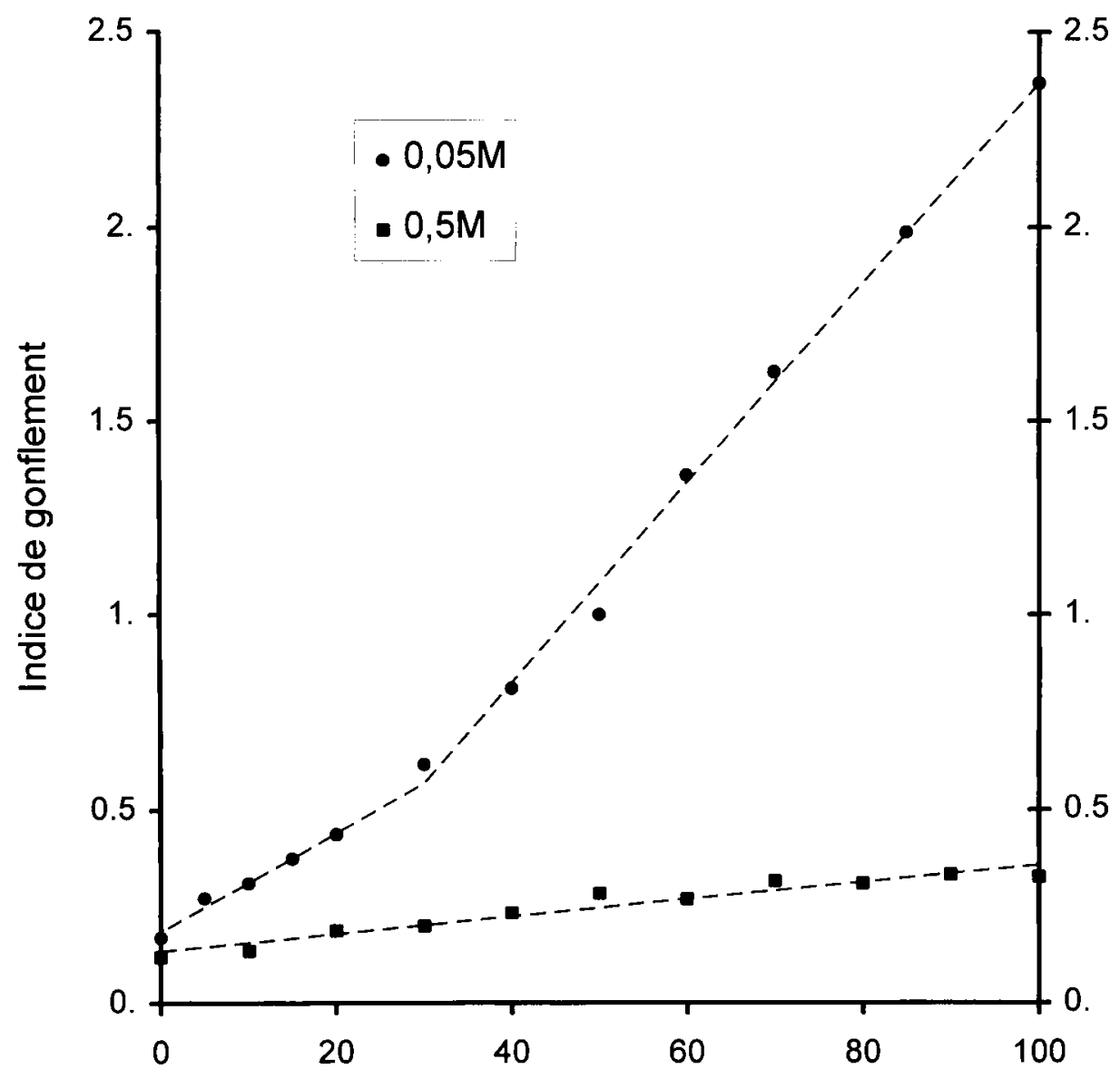

Fig 4. Relations indice de vide - Indice de retrait sous une sollicitation passant de 3,16 à $10 \mathrm{kPa}$ pour les mélanges consolidés à $100 \mathrm{kPa}$. 


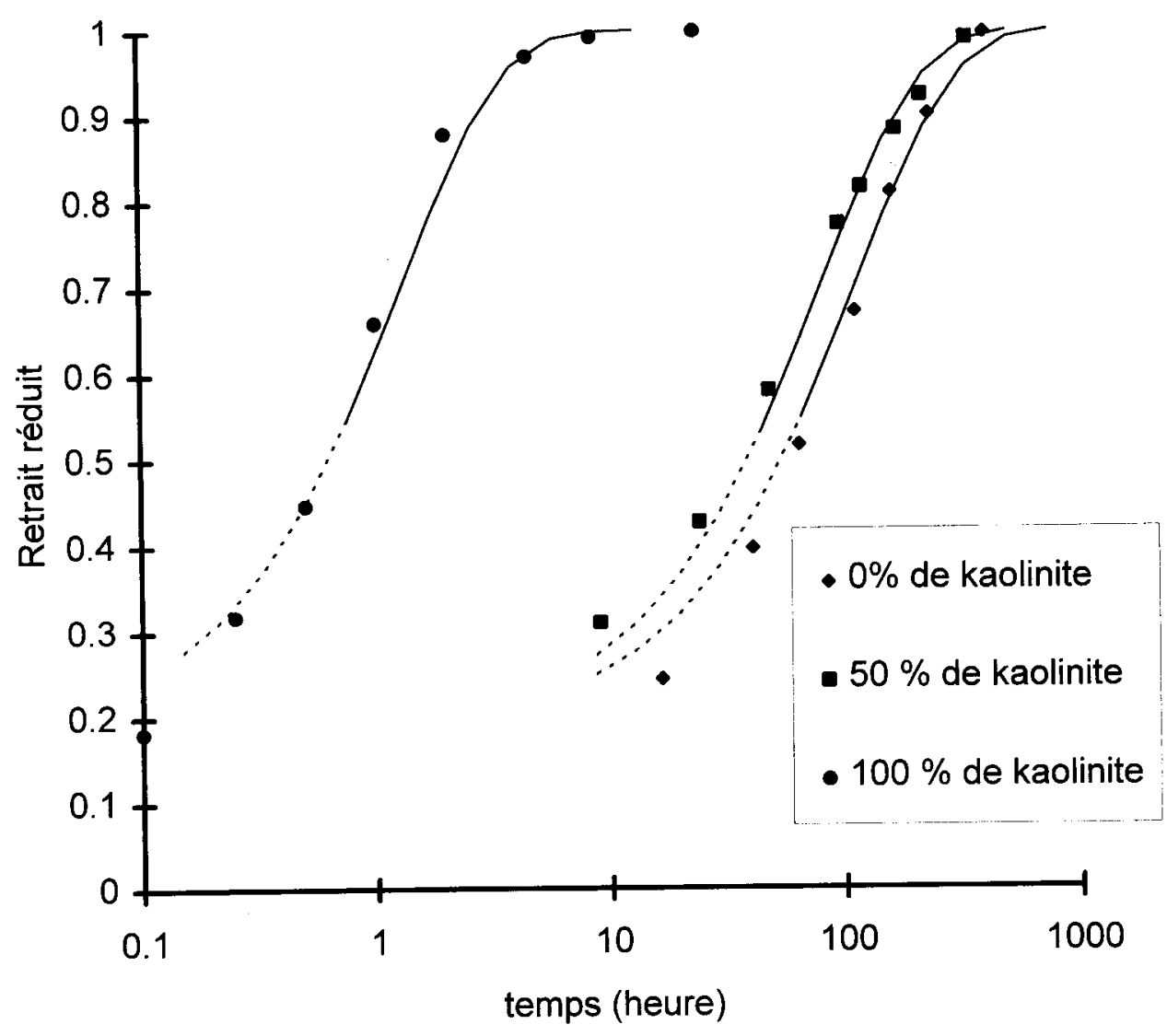

Fig 5. Évolution du retrait réduit en fonction du temps pour les mélanges argileux à $0,05 \mathrm{M}$.

Fig 6. Évolution du retrait réduit en fonction du temps pour les mélanges argileux à $0,5 \mathrm{M}$.

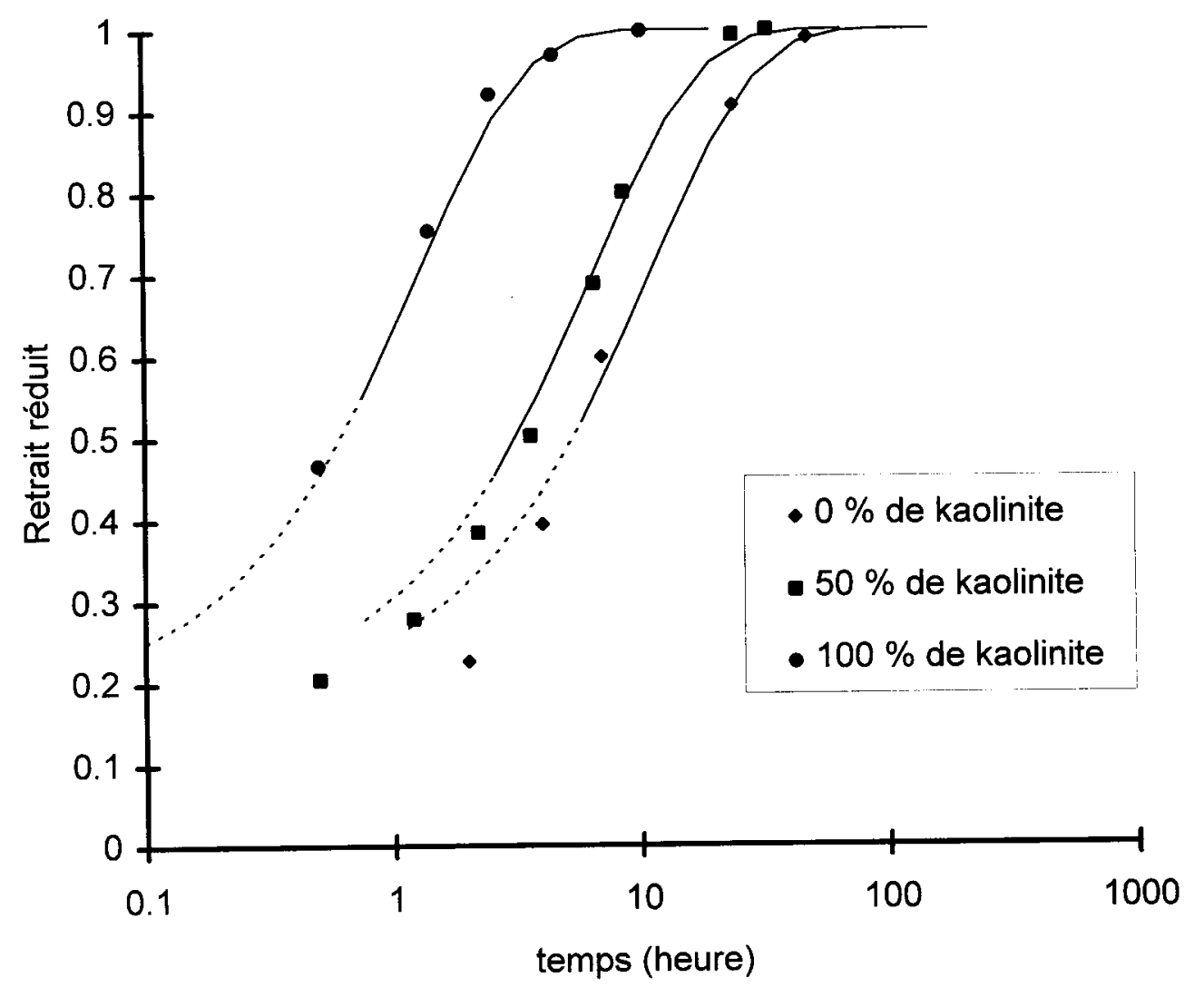


- la dizaine d'heures pour les échantillons ne contenant que de la kaolinite, quelle que soit la concentration de la solution ;

- la centaine d'heures pour les échantillons de montmorillonite à $0,5 \mathrm{M}$;

- le millier d'heures pour les échantillons de montmorillonite à $0,05 \mathrm{M}$.

Pour les opérations de gonflement, les valeurs expérimentales sont bien ajustées avec l'équation [14] puisque toutes les relations étudiées ont des $r^{2}$ supérieurs à 0,96 . Les hypothèses retenues pour l'élaboration de ces dernières peuvent donc être considérées comme valables avec des déformations faibles en gonflement-retrait et une perméabilité intrinsèque constante. Nous avons choisi de déterminer $\mathrm{Cv}$ puis $\mathrm{k}$ pour chaque échantillon à partir des régressions obtenues sur les points correspondants à $t_{\mathrm{R}}>0,2$. Pour chaque suspension, le coefficient de régression b est égal à :

$$
\mathrm{b}=\frac{\pi^{2} \cdot \mathrm{C}_{\mathrm{v}}}{4 \cdot \mathrm{l}^{2}}=-\frac{\pi^{2} \cdot \mathrm{k} \cdot(1+\mathrm{e})}{4 \cdot \mathrm{l}^{2} \cdot \mathrm{a}_{v} \cdot \eta}
$$

Il est possible de calculer $k$ pour tous mélanges étudiés, à partir des étapes suivantes : le coefficient de compressibilité $a_{v}$ est déterminé avec la formule [2] au point d'abscisse 5,6 $\mathrm{kPa}$; la valeur de e retenue est la valeur moyenne de l'indice de vide pendant l'opération de retrait ; 1 est calculée à partir du volume de l'échantillon; la valeur de la viscosité de l'eau $\eta$ est prise à $20^{\circ} \mathrm{C}$ soit 0,001 Pa.s.

Les résultats de ces différents traitements sont reportés sur la figure 7 . On y observe que :

- pour les milieux à $0,5 \mathrm{M}$, la perméabilité décroît régulièrement de $2,56 \quad 10^{-6} \mathrm{~m}^{2}$ à $0,9410^{-16} \mathrm{~m}^{2}$ avec l'augmentation de la teneur en montmorillonite ;

- avec les mélanges obtenus à $0,05 \mathrm{M}$, la baisse de perméabilité est plus importante et varie de $2,3410^{-16} \mathrm{~m}^{2}$ à $0,3310^{-16} \mathrm{~m}^{2}$. Cette dernière valeur obtenue sur la montmorillonite est très voisine de la valeur de $0,2 \quad 10^{-16} \mathrm{~m}^{2}$ obtenue par Kirby et Smiles (1988) sur la même montmorillonite du Wyoming pour une charge hydraulique de $-10 \mathrm{~J} / \mathrm{kg}(10 \mathrm{kPa})$. Cette proximité témoigne de la validité du paramètre de perméabilité avec une excellente convergence des résultats obtenus à partir d'approches différentes (relaxation de contrainte et filtration). Cependant, cette chute est brutale aux basses teneurs en montmorillonite, puisque dès $40 \%$ la perméabilité a déjà atteint
Fig 7. Relations perméabilitéteneur massique en montmorillonite pour les mélanges argileux consolidés à $100 \mathrm{kPa}$.

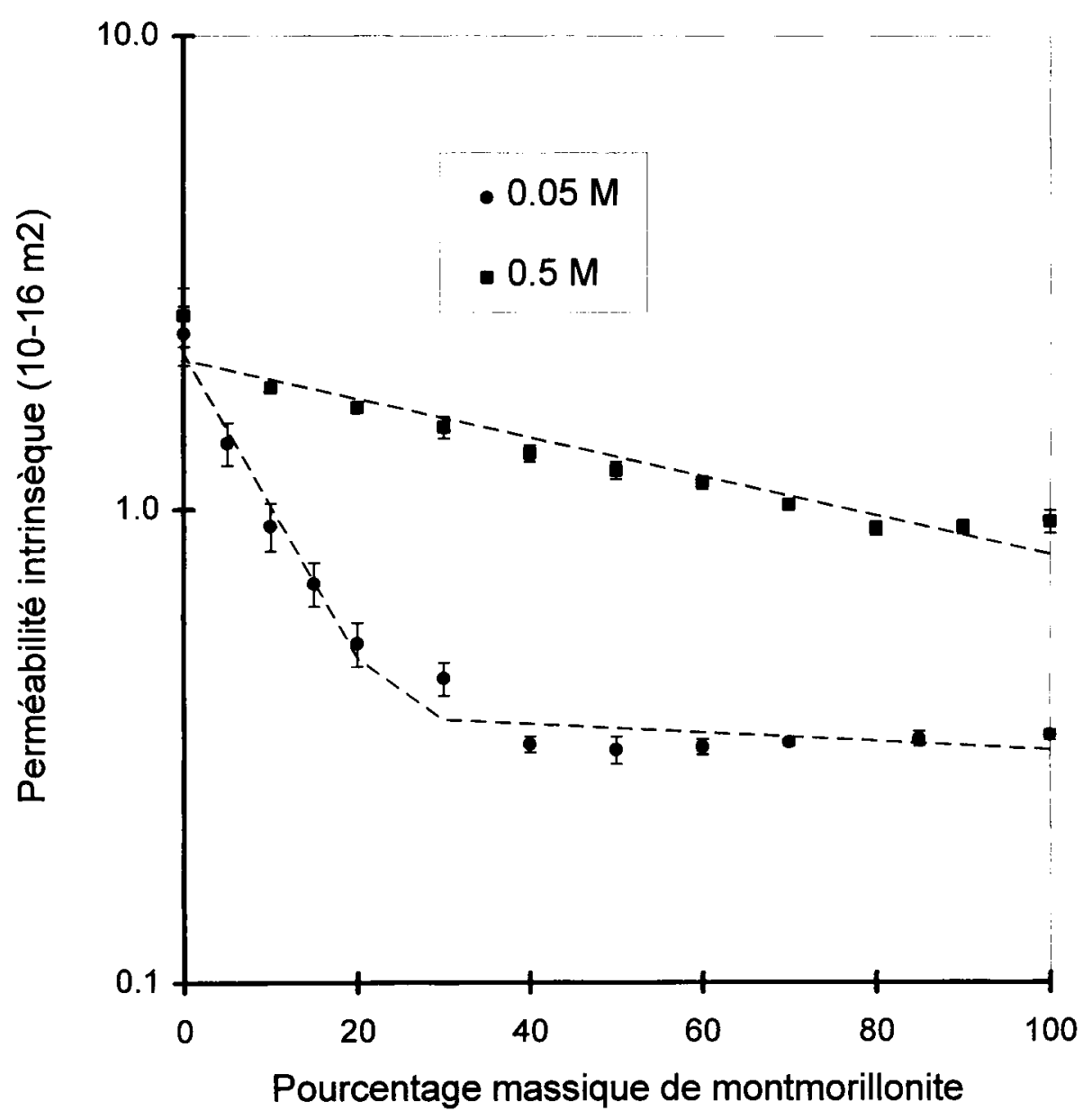


$0,3210^{-16} \mathrm{~m}^{2}$. À l'image des deux autres paramètres étudiés, le seuil observé à $30 \%$ pour les milieux à $0,05 \mathrm{M}$ est aussi perceptible sur la perméabilité.

\section{Analyse d'images obtenues au microscope à balayage}

Au-delà d'une interprétation sur l'organisation interne d'un milieu à partir de l'étude de paramètres mesurés macroscopiquement de l'extérieur, il est tentant de vouloir vérifier ces résultats par une observation directe de cette organisation. Des images au microscope à balayage ont été réalisées sur les échantillons $0,05 \mathrm{M}$ à 20,30 et $40 \%$ de montmorillonite, mélanges qui se situent autour du seuil observé. Les prises de vues obtenues pour les trois milieux sont regroupées sur la figure 8. La largeur des clichés représente $60 \mathrm{~mm}$. Sur le mélange à $20 \%$ de montmorillonite, on distingue encore très bien les cristallites de kaolinite dont les dimensions, si l'on fait abstraction des quelques grosses particules, sont voisines de 1 à 2 micron. Pour les deux autres échantillons, les cristallites sont à peine visibles et le réseau « filamenteux », caractéristique des montmorillonites devient prépondérant à $30 \%$ et totalement généralisé à $40 \%$. Les vides du réseau ont alors des dimensions qui vont de 2 à $4 \mathrm{~mm}$. En retenant l'hypothèse d'une porosité interparticulaire constante pour les trois échantillons et égale à $59 \%$, les images ont été seuillées arbitrairement à cette valeur pour discriminer les surfaces claires (solide) des surfaces foncées (porosité). Les particules obtenues avec ce seuillage ont été labellisées et leur surface calculée. La figure 9 montre la variation de la médiane des cent plus grosses particules et met en évidence le passage, pour les trois échantillons étudiés, d'un milieu à particules de porosité de grandes dimensions vers un milieu à particules de porosité de petites dimensions. Corrélativement, les particules de solide suivent une évolution inverse avec la mise en évidence d'un réseau solide trés connecté. Ces considérations sont illustrées sur les images de la figure 10 où sont représentées les plus grosses particules de solide et de porosité dans les mélanges à 30 et $40 \%$.

\section{DISCUSSION}

Largement initiée par les travaux de Fiès et al (1972), l'étude de la porosité a surtout porté sur l'observation du comportement de mélanges de familles de particules aux diamètres très contrastés : les unes étant sensées jouer le rôle du squelette et les autres celui du plasma. Pour les mélanges étudiés ici on peut, à partir des travaux de Tessier (1984), rappeler la configuration des particules qui les composent. Si toutes les particules ont des épaisseurs voisines de $0,1 \mathrm{~mm}$, c'est essentiellement par leur organisation interne et leur extension latérale qu'elles diffèrent :

- bien cristallisés et anhydres avec une extension latérale de $1 \mathrm{~mm}$ pour les cristallites de kaolinite ;

- bien cristallisés mais avec deux à trois couches de molécules d'eau entre les feuillets et une extension latérale de $2 \mathrm{~mm}$, voire $5 \mathrm{~mm}$ pour les quasicristaux de smectite ;

- empilement turbostatique et extension latérale toujours inférieure à $0,5 \mathrm{~mm}$ pour les tactoïdes mais avec la possibilité de s'associer bord à bord pour former des réseaux de grande extension.

Pour les mélanges à $0,5 \mathrm{M}$, les valeurs d'indices de vide et de gonflement sont situées sur une ligne qui joint les deux pôles (kaolinite pure et montmorillonite pure). Avec des particules de dimensions voisines, cette non-interaction peut facilement s'expliquer par la substitution de cristallites par des quasicristaux dans les arrangements. L'augmentation de l'indice de vide s'expliquerait ainsi par la substitution de particules anhydres (cristallites) par des particules possédant des couches d'eau intercalaire (quasicristaux). Si l'on suppose que la porosité interparticulaire reste constante et égale à 1,41 (valeur obtenue pour $100 \%$ de kaolinite), l'indice de vide intraparticulaire à affecter aux quasicristaux pour obtenir l'augmentation d'indice de vide accompagnant l'augmentation du taux de montmorillonite est de 0,84 . Cette valeur est très proche du résultat obtenu à partir de quasi-cristaux de montmorillonite hydratés à trois couches d'eau qui présentent une distance interfoliaire de $1,86 \mathrm{~nm}$, valeur observée par Tessier (1984). La légère baisse de perméabilité observée avec l'augmentation de la teneur en montmorillonite ne pourrait alors s'expliquer que par une modification des propriétés à l'interface solide-eau. S'il est donc possible de retenir un modèle de non interaction pour les mélanges $0,5 \mathrm{M}$, il reste délicat de tenter d'appliquer les modèles proposés par les auteurs cités précédemment aux autres mélanges testés. L'interaction observée pour les mélanges à $0,05 \mathrm{M}$ peut difficilement être attribuée à un phénomène de remplissage de quelques vides de la kaolinite par des particules de smectites. Elle pourrait en revanche être liée au remplacement 


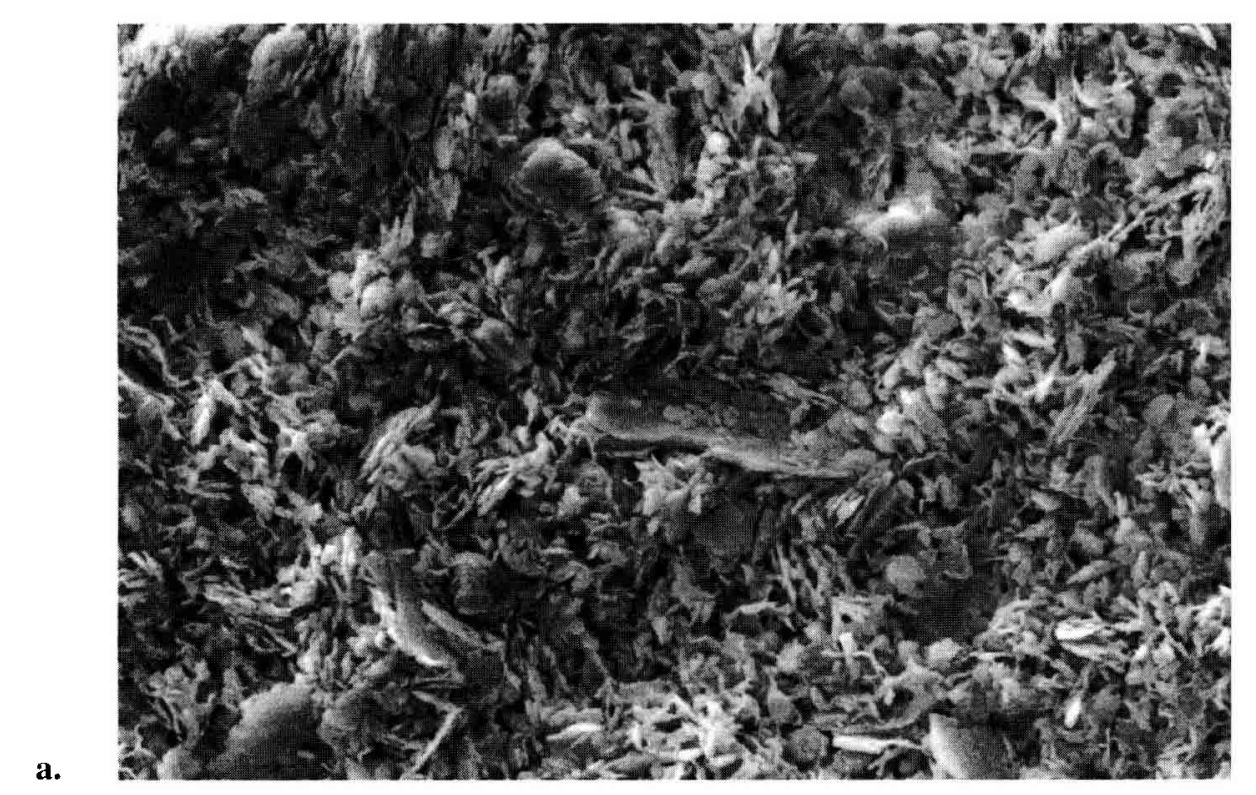

b.
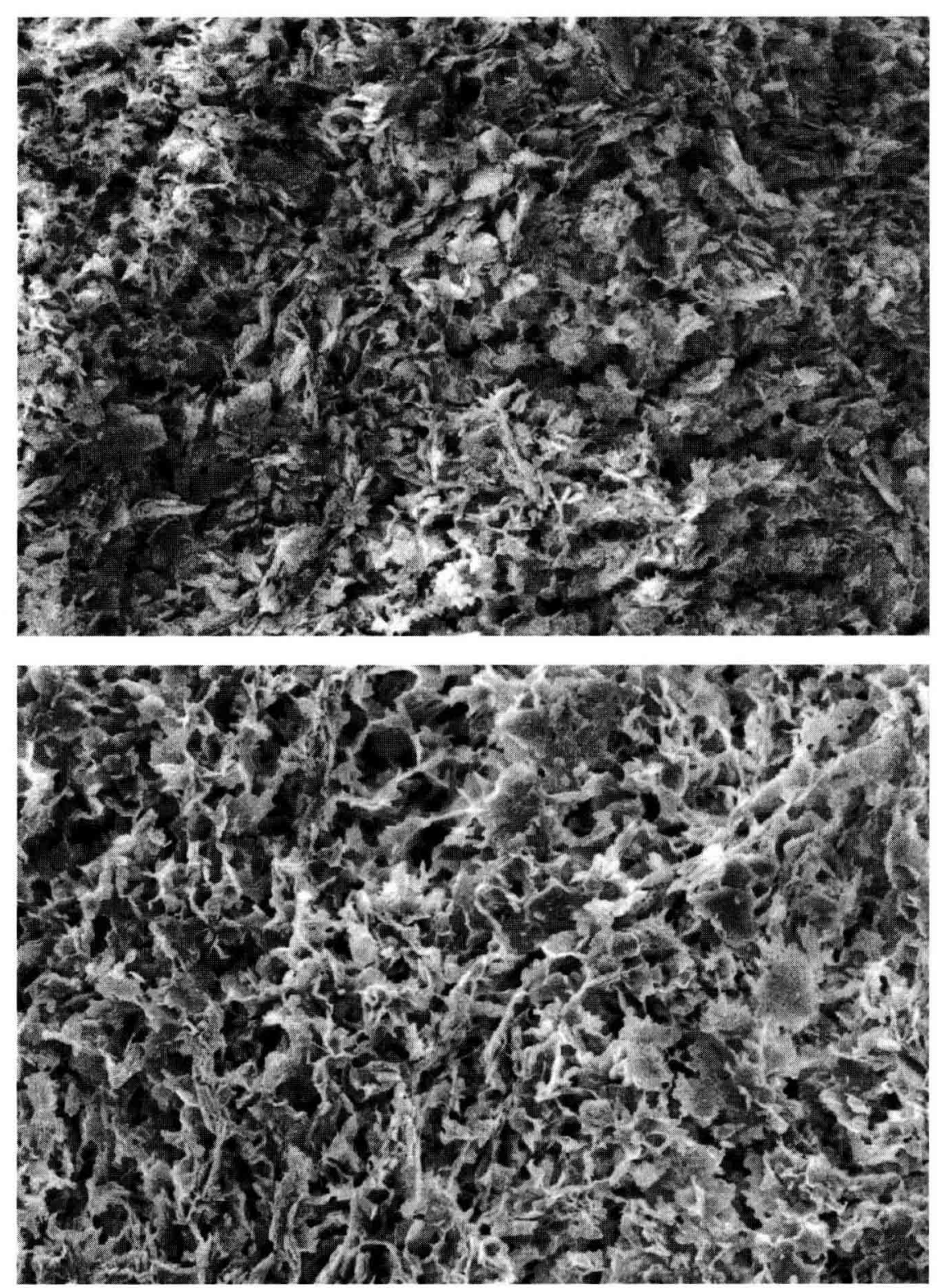

Fig 8. Microphotographies (largeur du cliché : $60 \mu \mathrm{m}$ ) des mélanges à a. $20 \%$ de montmorillonite. b. $30 \%$ de montmorillonite, c. $40 \%$ de montmorillonite. 


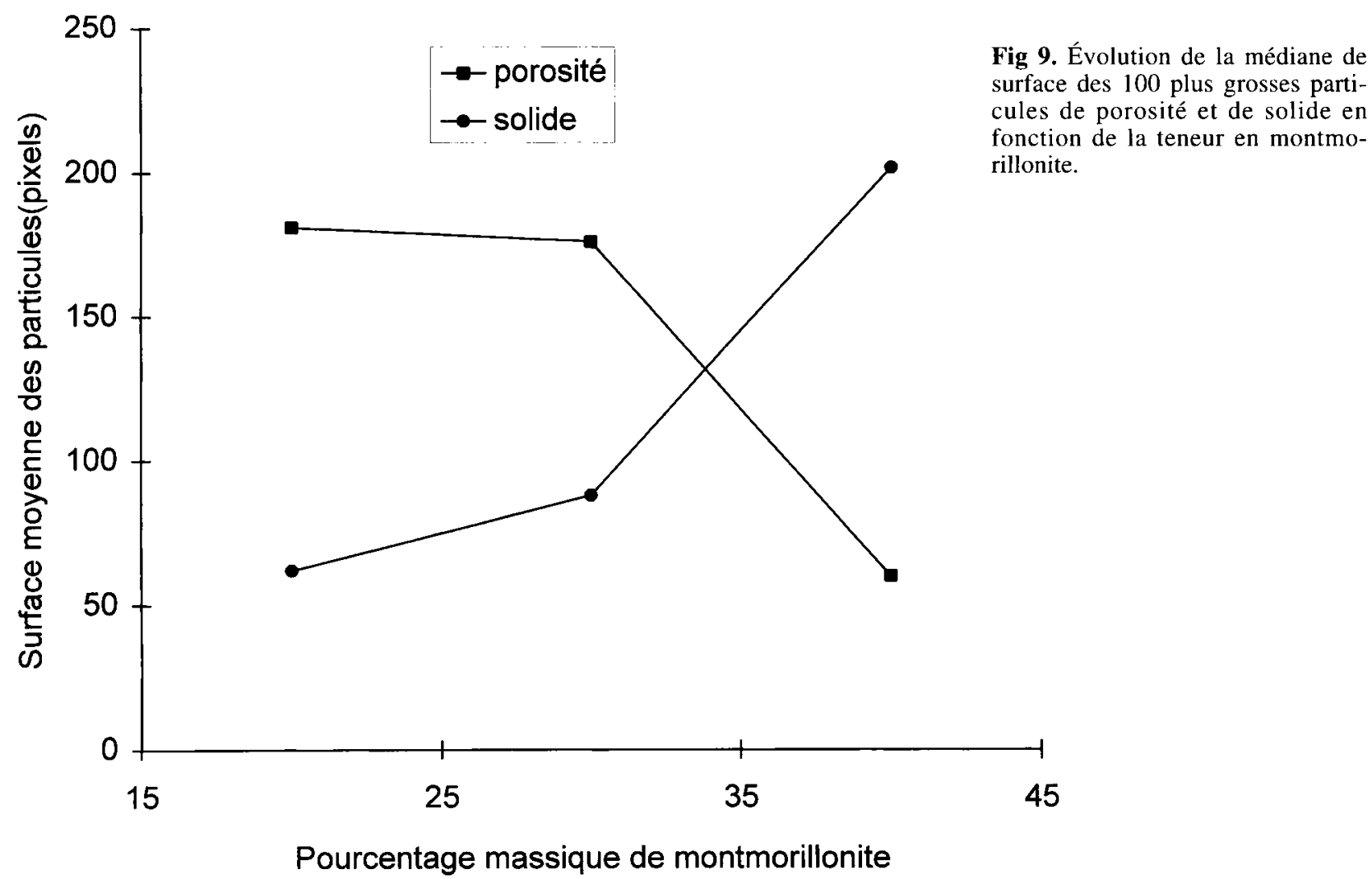

dans les empilements de quelques particules de kaolinite par des particules de smectite plus petites mais surtout aux formes moins aplaties donc génératrices d'une porosité plus faible, et aussi plus flexibles donc susceptibles de se déformer en fonction de la porosité générée par l'assemblage des cristallites. Tant que leur pourcentage reste faible, on peut supposer que la probabilité que deux particules de tactoïdes se côtoient reste faible. Lorsque leur densité augmente dans le milieu, les possibilités d'association bord à bord augmentent aussi et la constitution d'un réseau continu d'extension importante deviendrait effective lorsque le taux de montmorillonite atteindrait $30 \%$.

Une hypothèse complémentaire à la précédente peut être reprise pour avancer que jusqu'à cette teneur, l'assemblage des cristallites entre eux reste prépondérant pour la résistance du milieu à la déformation. Au-delà de cette valeur, les propriétés gonflantes de la montmorillonite s'expriment proportionnellement aux quantités introduites. D'autres résultats mettaient déjà en évidence ce seuil pour des propriétés physiques comme la résistance au cisaillement. En effet, les mélanges montmorillonite sodique-quartz 60-2 mm utilisés par Kenney (1967, in MüllerVonMoos et Loken, 1989) et les mélanges bento- nite sodique-kaolinite de Di Maio et Fenelli (1994) atteignent aussi le minimum de résistance au cisaillement dès que la teneur en smectite atteint $30 \%$. Comment ne pas aussi faire état des résultats obtenus par Fiès (1984) en porosimétrie sur des mélanges sable-argile d'une part et limon argile d'autre part. Pour les premiers la porosité d'accès restait constante jusqu'à $30 \%$ d'argile et pour les seconds jusqu'à $20 \%$.

À partir d'une teneur voisine de $30 \%$ de montmorillonite, la perméabilité intrinsèque du mélange argileux a fortement chuté et est totalement déterminée par celle de la montmorillonite pure. On peut donc concevoir qu'à partir de ce seuil de $30 \%$, l'eau en mouvement ne peut plus éviter un passage par une porosité définie par la montmorillonite. Ces voies de circulation entre pores de grande taille dans les smectites sous forme de tactoïdes se font toujours entre deux feuillets séparés par quelques dizaines de nanomètre d'eau (Halitim et al, 1984). Au-delà du seuil de $30 \%$, les propriétés hydrodynamiques des smectites domineraient dans l'ensemble du massif. L'hypothèse d'un artefact expérimental lié à une chute de perméabilité importante au contact filtre-échantillon a souvent été émise dans le cas des mesures hydrodynamiques sur colonne de sol reposant sur un filtre. Elle peut être en grande partie levée 
a.

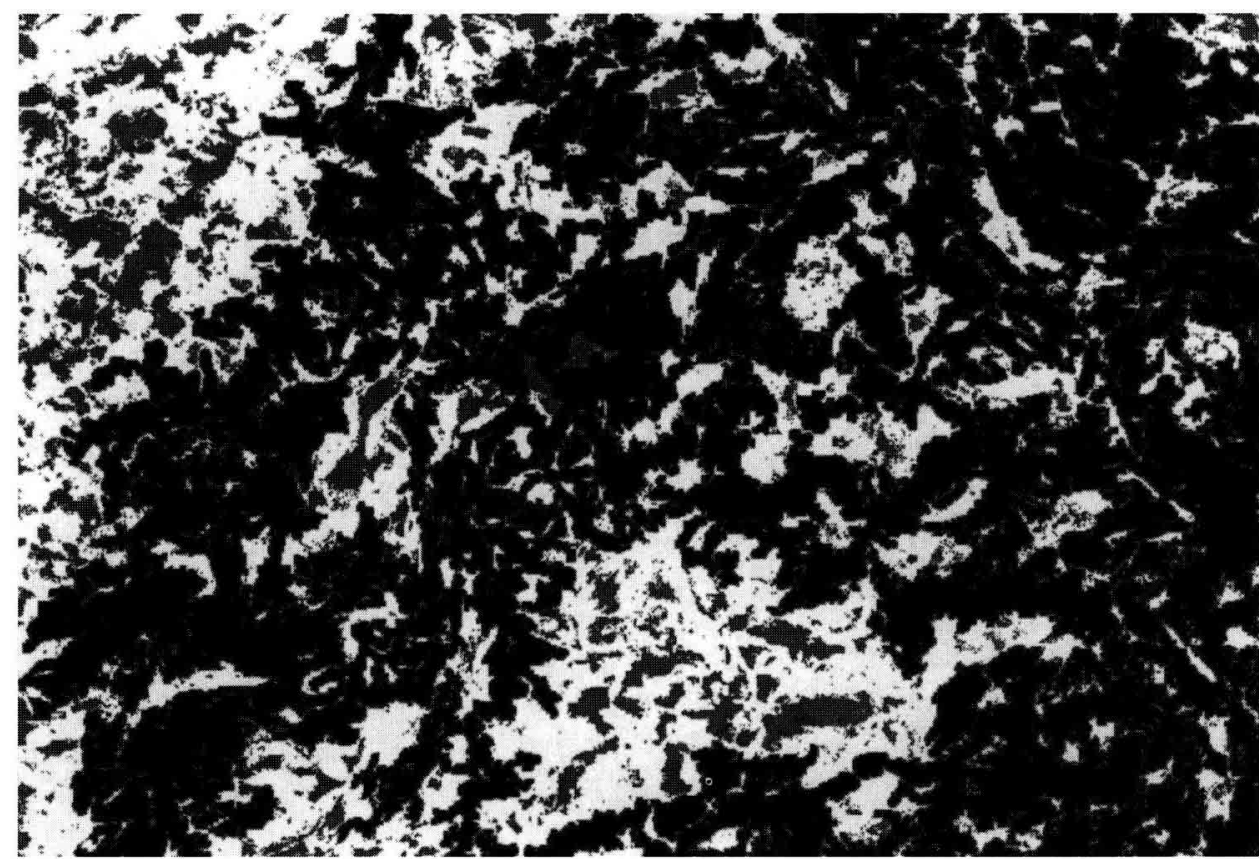

b.

Fig 10. Seuillage et labellisation de la porosité à a. $30 \%$ de montmorillonite b. $40 \%$ de montmorillonite.

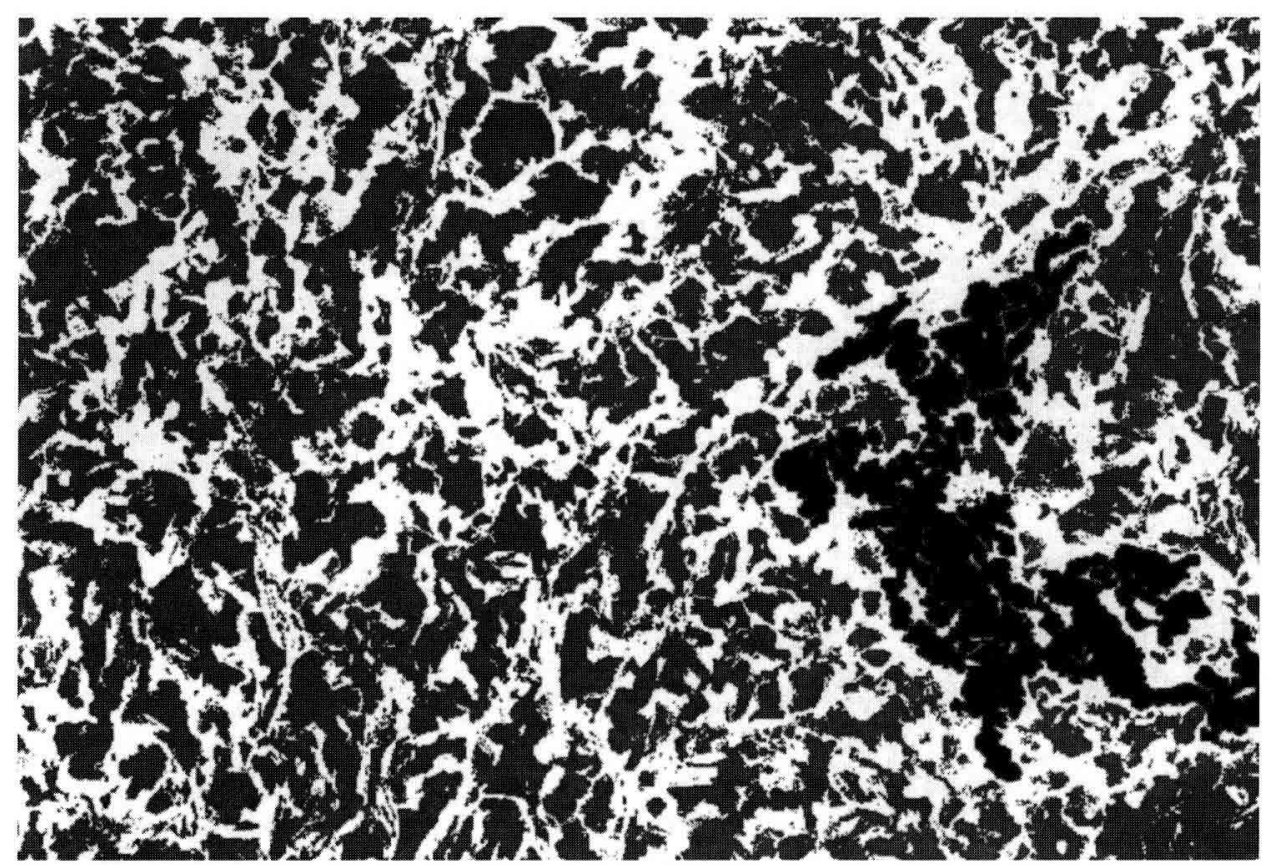

dans la mesure où les résultats obtenus sur les indices de vide et de gonflement sont des mesures à l'équilibre qui concernent la totalité de l'échantillon. Pour ces paramètres, le seuil de $30 \%$ est parfaitement observable dans les milieux à $0,05 \mathrm{M}$.

Même si elles doivent être utilisées avec précaution, le traitement des quelques observations microscopiques met en évidence une modification de la structure de l'échantillon. On peut en effet supposer que la fracture de l'échantillon congelé s'est réalisée selon une surface qui privilégiait le contenu en montmorillonite dont la résistance au cisaillement est plus faible : le fait que le réseau apparaisse comme quasiment continu lorsqu'on passe de 30 à $40 \%$ de montmorillonite conforte l'hypothèse proposée plus haut avec un réseau de tactoïdes fortement connecté et un transfert hydrique qui ne peut trouver de chemin préférentiel évitant un passage entre les particules de montmorillonite. 


\section{CONCLUSION}

Nos résultats ont mis en évidence un seuil de concentration en montmorillonite à partir duquel leurs propriétés hydrodynamiques en conditions diluées et sodiques s'imposent à la totalité du milieu où elle sont présentes. Ce seuil est important car s'il est souvent atteint par les smectites au sein de la fraction argileuse, il l'est quelquefois aussi au niveau de la fraction fine des sols. La présence fréquente dans les sols de minéraux interstratifiés associant des minéraux de type gonflant et non gonflant pourrait aussi induire une sensibilité aux conditions physicochimiques dès les faibles teneurs. La sensibilité exacerbée de certains sols aux conditions hydriques et physicochimiques s'expliquerait alors plus facilement.

\section{REMERCIEMENTS}

L'auteur remercie J Berrier de l'Inra de Rennes pour l'acquisition des images microscopiques.

\section{RÉFÉRENCES}

Bradford JM, Gupta SC (1986) Compressibility. In: Methods of Soil Analysis: Part I, Physical and Mineralogical Methods, 2nd edn (A Klute, ed), Am Soc of Agronomy, Madison, WI, 479-492

Cabidoche YM, Voltz M (1995) Non-uniform volume and water content changes in swelling clay sol: II. A field study on a vertisol. Eur J Soil Sci 46, 345356

Cases J, François M (1982) Étude des propriétés thermodynamiques de l'eau au voisinage des interfaces. agronomie 2, 931-938

Cases JM, Bérend I, Besson G, François M, Uriot JP, Thomas F, Poirier JE (1992) Mechanism of adsorption and desorption of water vapor by homoionic montmorillonite. 1. The sodium-exchanged form. Langmuir 8, 2730-2739

Charpentier S (1991) Déformation de matériaux argileux soumis à une contrainte mécanique en conditions saturées. Influence de la concentration et de la nature des solutés. Thèse Ensar, Rennes

Chenu C, Tessier D (1995) Low temperature scanning electron microscopy of clay and organic constituents and their relevance to soil microstructure. Scanning microscopy 9, 989-1010

Costet J, Sanglerat G (1981) Cours pratique de mécanique des sols. Bordas, Paris, $283 \mathrm{p}$

Cuisset O (1979) Le potentiel électrocinétique des argiles. Influence de la salinité. Bull de Liaison des Laboratoires des Ponts et Chaussées 104, 15-20
Di Maio C, Fenelli GB (1994) Residual strength of kaolin and bentonite: the influence of their constituent pore fluid. Geotechnique 44, 217-226

Fiès JC (1984) Analyse de la répartition du volume des pores dans les assemblages argile-squelette : comparaison entre un modèle d'espace poral textural et les données fournies par la porosimétrie au mercure. agronomie 4, 891-899

Fiès JC, Hénin S, Monnier G (1972) Étude de quelques lois régissant la porosité de matériaux meubles. Ann Agron 23, 621-653

Gras R (1988) Physique du sol pour l'aménagement. Masson, Paris, $588 \mathrm{p}$

Halitim A, Robert M, Tessier D, Prost R (1984) Influence des cations échangeables $\left(\mathrm{Na}^{+}, \mathrm{Ca}^{++}\right.$, $\mathrm{Mg}^{++}$) et de la concentration saline sur le comportement phyique (rétention en eau, conductivité hydraulique) de la montmorillonite. agronomie 4, 451-459

Houpeurt A (1975) Éléments de mécanique des fluides dans les milieux poreux. Technip, Paris, $240 \mathrm{p}$

Keren R (1989) Effects of clay charge density and adsorbed ions on the rheology of montmorillonite suspension. Soil Sci Soc Am J 53, 25-29

Keren R, Singer MJ (1988) Effect of low electrolyte concentration on hydraulic conductivity of sodium/calcium montmorillonite - sand system. Soil Sci Soc Am J 52, 368-373

Kirby JM, Smiles DE (1988) Hydraulic conductivity of aqueous bentonite suspensions. Aust J Soil Res $26,561-574$

Low PF (1976) Viscosity of interlayer water in montmorillonite. Soil Sci Soc Am J 40, 500-505

Mamy J (1968) Recherches sur l'hydratation de la montmorillonite : propriétés diélectriques et structures du film d'eau. Ann Agron 19, 175-292

Mieussens C, Magnan JP, Soyez B (1985) Essai de compressibilité à l'œdomètre. Bull de Liaison des Laboratoires des Ponts et Chaussées 139, 5-18

Müller-VonMoos M, Loken T (1989) The shearing behaviour of clays. App Clay Sci 4, 125-142

Norrish K, Quirk JP (1954) Crystalline swelling of montmorillonite - Use of electrolytes to control swelling. Nature 173, 255-256

Quirk JP, Schofield RK (1955) The effect of electrolyte concentration on soil permeability. J Soil Sci 6, $163-178$

Tessier D (1984) Étude expérimentale de l'organisation des matériaux argileux. Hydratation, gonflement et structuration au cours de la dessiccation et de la réhumectation. Thèse, université Paris-VII, $361 \mathrm{p}$

Vauclin M (1987) Hydrodynamique dans les sols partiellement saturés déformables. Conférence de formation continue, Institut de mécanique, Grenoble, $41 \mathrm{p}$ 